\title{
Responsible Human-Robot Interaction with Anthropomorphic Service Robots: State of the Art of an Interdisciplinary Research Challenge
}

\author{
Ruth Stock-Homburg ${ }^{1}$, Jérôme Kirchhoff ${ }^{4}$, Judith S. Heinisch ${ }^{2}$, Andreas Ebert ${ }^{3}$, Philip Busch ${ }^{1}$, Niyati Rawal ${ }^{1}$, \\ Klaus David ${ }^{2}$, Janine Wendt ${ }^{1}$, Indra Spiecker gen. Döhmann ${ }^{3}$, Oskar von Stryk ${ }^{4}$, Martin Hannig ${ }^{1}$, and Merlind Knof ${ }^{1}$ \\ ${ }^{1}$ Department of Law and Economics, Technische Universität Darmstadt, \\ Email: $\{$ rsh,merlind.knof,niyati.rawal,martin.hannig\}@bwl.tu-darmstadt.de, $\{$ busch,wendt $\} @$ jus.tu-darmstadt.de \\ ${ }^{2}$ Department of Electrical Engineering and Computer Science, Universität Kassel, \\ Email: \{judith.heinisch,david $\} @$ uni-kassel.de \\ ${ }^{3}$ Department of Law, Goethe-Universität Frankfurt am Main, Email: \{ebert,spiecker\} @ jur.uni-frankfurt.de \\ ${ }^{4}$ Department of Computer Science, Technische Universität Darmstadt, Email:\{kirchhoff,stryk\}@ sim.tu-darmstadt.de
}

\begin{abstract}
Anthropomorphic service robots are on the rise. The more capable they become and the more regular they are applied in real-world settings, the more critical becomes the responsible design of human-robot interaction (HRI) with special attention to human dignity, transparency, privacy, and robot compliance. In this paper we review the interdisciplinary state of the art relevant for the responsible design of HRI. Furthermore, directions for future research on the responsible design of HRI with anthropomorphic service robots are suggested.
\end{abstract}

\section{Introduction}

In the last decade, anthropomorphic service robots with extremities such as arms, legs or a head [1], autonomous behavior and the use of artificial intelligence (AI) in service provision have offered useful perspectives (see $[2,3]$ for overviews). Automatic teller machines, self-checkout machines, and touchscreen ordering of fast food are well-established technologies and are precursors to service robots. Although robotic technology is still emerging, "human-humanoid encounters in the marketplace are not as futuristic as they might seem, and they represent a primary arena for innovation in services" [4, p. 535]. Service robots will support humans by providing various services, e.g., information and advice in several areas, such as banking, retail, hospitality, health, and education [5,6].

In contrast to digital or other robotic systems, anthropomorphic robots can express emotion-like signals through gestures, facial expressions, and haptics (i.e., hand contacts, touch). This allows human users to interact with anthropomorphic robots, also, through nonverbal communication [3] and even build connections [7]. Physical presence is a key success factor for the goals of human-robot interaction (HRI) and unleashing the potential of anthropomorphic service robots [8]. In this paper, HRI is defined as "the study of the humans, robots, and the ways they influence each other" $[9$, p. 256]. To integrate these unique robots in our lives, it is important to address ethical issues now in a responsible design of HRI, its mechanisms of action, and its control. "If we do not address futuristic ethical issues now, it may be too late once technological innovations force them upon us" [10, p. 2].

Our interdisciplinary research project "RoboTrust" pursues the scientific guiding idea of responsible design of anthropomorphic service robots. To this end, we elaborated the following premises for our research on anthropomorphic service robots. Hence, these robots (a) support (and do not replace) the human in areas that the human cannot perceive due to resource constraints (see Section 2.1); (b) consider human dignity and act in an, e.g. emotionally, responsive manner (see Sections 2.1 and 2.5); (c) are transparent and predictable in their interaction behavior (see Section 2.4); (d) transparently collect and process data in accordance with applicable data protection laws (see Section 2.3); and (e) fall under a clearly established liability regime.

Thus, we define the term responsible design as the design of HRI between anthropomorphic service robots and users with special attention to human dignity, emotional needs, privacy \& data protection, transparency, and safety \& security. This basic notion of "RoboTrust" allows the development of 
anthropomorphic service robots in a way that enables HRI that is authentic and acceptable to humans. This leads, among others, to the necessity of applying adequate measures in order to evaluate the psychological needs of participants of HRI experiments (see [11] for an overview).

Various disciplines contribute to HRI leading to the interdisciplinary character of this research area, notably ethics, law, psychology, and technology [12]. However, to the best of the authors' knowledge the state of the art regarding HRI involving anthropomorphic service robots is incomplete, as it remains on an abstract level, covers the requirements only partially or does not provide a comprehensive approach [13-17]. This is especially true, if the interdisciplinarity of designing HRI responsibly is taken into account. Therefore, we identified the need for a state of the art review concerning the interdisciplinary research, relevant for the responsible design of HRI with anthropomorphic service robots covered by "RoboTrust" (see Section 2). Additionally, the interdependencies of the research disciplines involved are considered (i.e., ethics, law, psychology, and technology, see Section 3). Finally, areas for future research are identified and suggested based on the gained knowledge (see Section 3).

\section{State of the Art on Responsible HRI}

\subsection{Ethics in HRI}

Extant literature has discussed several ethical concerns [10]: ubiquitous surveillance, such as monitoring and storage of personal and behavioral digital data [18]. Interfaces, such as Apple's Siri, Amazon's Alexa, Microsoft's Cortana, and Google Home "not only listen to what we say, they also record it and send it back to Amazon, Apple, or Google" [10, p. 3]. This brings up issues of compliance (see Section 2.2) and privacy (see Section 2.3) but also aspects of human dignity in that users have been shown to feel disgust towards such conversational digital agents [7, 19]. Against this background, ethical considerations related to HRI have entered academic and philosophical discussions in various disciplines, such as machine ethics [20], computer ethics [21], and robot ethics [22]. In 2019 a complementary "Ethical Aligned Design" has been proposed for autonomous and intelligent systems [23]. Until today, there is an ongoing discussion as to whether ethical robots are possible or even desirable. This section will provide a brief overview on the definitions of robotic ethics, its theoretical foundations, and the current state of the art related to anthropomorphic service robots.
The term robotic ethics (often termed as "RoboEthics" [22]) has at least three different meanings [24]: (a) the professional ethics of robotics, (b) the moral code programmed into the robots themselves, and (c) "the self-conscious ability to do ethical reasoning by robots-to a robot's own, self-chosen moral code" [24, p. 35]. The project "RoboTrust" focuses on the responsible design of HRI with anthropomorphic service robots. We therefore define robotic ethics as the moral code programmed into the robots themselves with a special attention to protect human dignity, transparency, privacy, and security.

Three branches of ethics are important to structure existing research in RoboEthics [25]: meta-ethics, applied ethics, and normative ethics. Meta-ethics is rather abstract and theoretical and focuses on the question whether a robot can act in a good or bad manner $[26,27]$ or could be held responsible for its actions [28]. Applied ethics focuses on moral guides to specific domains and examine which specific moral dilemmas may arise from the application of robots [29]. An important question here is: How should humans design, deploy, and treat robots in a particular situation [30]? Finally, normative ethics reflects aspects of the robot application that are obligatory, allowed, or forbidden. From this perspective, moral mediation theory of technology $[31,32]$ argues that due to the increased closeness between humans, humans' moral perceptions and decisions are increasingly technologically mediated. Furthermore, research on the service dominant logic (S-D logic; [33]) identifies several general ethical principals that AI technologies should follow, such as transparency, reliability, and fairness (e.g., [34]). These theoretical insights advocate for a mindful integration of ethical values and principles into technologies [35-37].

Moral philosophy is different from moral psychology in that psychology attempts to understand the interplay between emotions (see Section 2.5) and reason as cause for individual moral decisions, the philosophical perspective focuses on the "ought" rather than on the "is" [38]. This paper has a largely comprehensive approach on applied robotic ethics. It attempts to integrate perspectives from RoboEthics and robotic psychology [12,39] for the sake of a responsible design of anthropomorphic service robots.

\subsection{RoboCompliance - Safety and Security in HRI}

Inherent with the improvement of the service robots usability through the use of AI, their capability to cause harm is potentially increased $[17,40]$. To reduce this hazard of anthropomorphic robots to a minimum is the 
focus of Robot Compliance (from here on termed as RoboCompliance, see also Section 2.3).

By RoboCompliance we refer to a robot - based on the generally accepted definition of product compliance as "a product conforming to rules, such as specifications, policies, standards, or laws" [41, p. 3] - that acts in a privacy-compliant manner [42] with the necessarily collected data and at the same time does give rise to fears of harm to third parties when AI is used [43]. Consequently, we define RoboCompliance as compliance with all regulations specifically relevant to intelligent anthropomorphic social robots, including those in data protection and liability law.

Comprehensible and fair liability rules for damage caused by robots play a central role in the establishment of this new technology: By means of transparency, they create predictability in dealing with worst-case scenarios that the user may fear and thus create the prerequisites for acceptance of the technology. Therefore, it is crucial to deal with worst-case scenarios of liability cases caused by robots already during their development process. This ensures that autonomy and safety can sensibly be weighed against each other [40].

How comprehensible and fair liability rules are to be designed in concrete terms must be developed in conjunction with several other disciplines, including above all robotic ethics (see Section 2.1). Their input is indispensable for the implementation of a generally accepted distribution of liability. Also crucial is technological transparency (see Section 2.4), which forms the basis for causality proofs. Data collection and processing (see Section 2.3) ensure safe movement and interaction with humans. Infringements of the General Data Protection Regulation (GDPR) through the use of a robot may lead to substantial penalties for robot users. Thus, finding a balance between innovation promotion, regulation and consumer protection by means of liability is a challenging task to be considered in an interdisciplinary way [44].

The increased damage potential of advanced robots is particularly due to the use of AI. This results in behavior that is not always predictable (see Section 2.4), which, in addition to the uncertainty in the actions of the robot itself, also leads to problems in proving causality ex post in the case of liability [17,45].

Within the framework of the existing regulations, strict liability, i.e., liability independent of responsibility for misconduct, would be the best option. However, besides some problems in the application, which also result from the low predictability, e.g., of complex software [46], a strict liability would run the risk of considerably inhibiting the development of AI. Therefore, from this perspective, a negligence-based liability would be more purposeful [47].

Finally, for all the recognized risks in establishing service robots, the big picture should not be lost from view: Even if individual risks and claims may not be ignored, the use of AI and robots, in sum, arguably brings a "net safety gain". It can be assumed that automation prevents more accidents than it causes $[47,48]$. Therefore, solutions that do not inhibit innovation must be sought. For example, a fund [46] or insurance solution [49] could be beneficial. The European Union (EU) Commission currently proposes a risk-based regulatory approach for the regulation of AI [50]: Particularly risky AI systems will be banned or strictly regulated as "high-risk". In addition to documentation and transparency obligations, risk management systems must also be used. AI must also be robust, secure and accurate. This establishes conditions that also facilitate the proof of causality. We agree with the risk-based regulatory approach. However, it also requires interdisciplinary cooperation as aimed by "RoboTrust", to be able to realistically assess the specific risk in the use of AI.

\subsection{Privacy and Data Protection in HRI}

The increasing diffusion of robots in all different kinds of social situations has raised questions with regards to how these machines may endanger human development and social dynamics through the use of personal information [51], e.g., from camera images, which may be necessary for their correct function or full performance. For the responsible design of HRI, the implementation of appropriate safeguards for privacy and data protection is thus indispensable.

In legal sciences, the concept of privacy has been the subject of scholarly debates for a considerable amount of time. Especially the concept in the EU and the United States (US) varies despite equal terminology. In the US, privacy is closely connected to trespassing, physical intrusion and that, in essence, no direct constitutional right to privacy exists. Privacy is mainly seen as "the claim of individuals ... when, how, and to what extent information about them is communicated to others" [52, p. 7].

In the EU, the regulation of privacy is closely connected with the notion of data protection [53], i.e., a close connection of control over personal data due to its effect on personality rights [54]. The EU's 2018 GDPR [55] so far constitutes the obvious benchmark for a privacy-compliant robot. The US and the EU approach may grow closer in the future as the GDPR has served as a blueprint for regulation in several other countries, such as Brazil and Japan, but foremost the Californian 
Consumer Privacy Act [56]. For the purposes of this paper, a robot which complies with the GDPR will be considered as privacy-compliant as the provisions are more demanding than the US notion [57].

Despite its significance, the requirements of GDPR-compliant robotics formulated in the literature are surprisingly few. The existing works neither reflect the new legal framework introduced by the GDPR [58] nor focus on other areas of robot use, e.g., health [59] or delivery [60] services. Others delve into the specifics of certain platforms, such as robots using cloud computing $[59,61]$. Some analyze at least some GDPR principles, e.g., transparency, in certain contexts [62].

Central research to rely on is the privacy-related evaluation of using sensory data, which cause concerns [63] on their own. These concerns arise in particular with anthropomorphic service robots, which interact with humans, and less so with mechanical, non-social robots [63]. Respondents worried most about their informational privacy, when manufacturers or operators of social robots used their data [64]. Hacking and stalking may also evoke considerable concerns, whereas physical privacy concerns may be less prevalent [64]. In this regard, groundwork has been done on responsible, GDPR-compliant video data processing, which includes analyses of potential technical solutions [65, 66].

While legal and political proponents argue for strong privacy protection, several studies indicate that individuals often mindlessly deal with privacy-related issues. Despite having privacy concerns when using technologies, they often still disclose personal data, also referred to as privacy paradox $[67,68]$. Initial studies indicate that this privacy paradox may also occur in real HRI, and is even more pronounced towards android robots than towards humanoid robots [69].

\subsection{Transparency in HRI}

With the ongoing technological evolution of robots, especially social and service robots, the number of possible applications for these intelligent or autonomous systems continues to grow. In situations where interaction with humans is a key factor for completing a task successfully and effectively, the development of these robots is faced with special challenges. The concept of transparency aims to support overcoming these challenges. In literature, transparency is described, amongst others, as offering explanations of actions [70], as the amount of information provided by the system to the user about internal operations [71], or as a design requirement to not exploit vulnerable users in a deceptive way [72]. In addition, transparency can be a means to enable trust in automated systems or robots
[73,74], but furthermore, its positive influence on human performance can be affected by the reliability of the specific robot [75].

Two prominent transparency models have been proposed: (1) for autonomous agents (e.g., robots) and (2) regarding HRI [76, 77]. The situation awareness-based agent transparency (SAT) model [76] consists of three levels representing different transparency aspects (i.e., task parameters, logic, and predicted outcomes), based on [78, 79] and [80]. It aims to provide a foundation for transparent interfaces that allow the operator to make informed decisions for interventions. The second model [77] distinguishes between robot-to-human and robot-of-human transparency. These transparency aspects group the factors into information concerning the robot (i.e., intention, task, analytics, environment) and the robot's awareness of the human (i.e., teamwork, human state), that needs to be provided. The SAT model and the robot-to-human transparency show some similarities regarding the contents conveyed.

Reviews on empirical studies that examine the effect of transparency [81,82] show that the literature mainly focused on the SAT model but the findings are incomplete or inconsistent, e.g., on the desirable level of transparency. Further, they argue that future research should investigate the appropriate transparency levels in relation to the context, why transparency supports the operator, and to use findings from cognitive psychology.

In the referenced literature the research focused primarily on the effects of transparency regarding an operator or teammate. Although many topics are still open here, the ethical and societal aspects are usually not considered. In [62] the transparency expectations for different stakeholders based on the work of [83] are shown and it is emphasized that legal transparency requires interdisciplinary collaboration among legal, social science, and technology. Further, they proposed a checklist to guide robot developers in implementing the GDPR's transparency requirements, which is, in particular, relevant to service robots processing personal data (see Section 2.3).

In order to establish measurable levels of transparency, the IEEE Standards Association is preparing a new standard "P7001 - Transparency of Autonomous Systems" [84]. It also considers different stakeholders to objectively determine the levels of compliance of an autonomous system, which goes conform with [62]. It also demands for technical solutions to investigate the causes, which may have led to malfunctions or incidents, and also to efficiently store with high integrity the data required for post mortem analysis [85]. Such a black box recorder for 
autonomous and AI driven robot behavior would help to track down causes of failure to the responsibility of hardware, software or user.

Transparency plays an important role in HRI and the research efforts on it form a solid basis but still show partly inconsistent findings and open issues. Further, an evidence-based interdisciplinary guideline of how to realize transparency in specific contexts is still missing. This also includes the case where transparency is potentially not desirable, e.g., for social robots [86].

\subsection{Evaluation of Responsible HRI}

Evaluation is " $[t]$ he process of determining the merit or worth or value of something" [87, p. 53]. With respect to HRI, it provides a systematic approach to assess to which extent the goal of responsible HRI is achieved. Extant research on the measurement of HRI can be organized in three categories: (1) (subjective) perceptual measures (i.e., self-perceptions, observer perceptions), (2) (objective) performance measures, and (3) sensor-based measures (e.g., psycho-physiological measures, face recognition measures) (see [88-91] for an overview).

Subjective perceptual measures can be conducted from a self- and observer perspective, using self-reported and behavioral indicators. Self-perceptions are based on subjective observations or feelings from a person. Often, they are not directly observable, but measurable through indicators, or identifiable via self-reports of human participants interacting with robots $[91,92]$. Psychometric scales, surveys, and questionnaires $[88,93]$ are suitable to assess trust [94, 95], acceptance [92, 95], and several participants' characteristics [90]. Contrarily, observer-perceptions investigate how observers understand behavioral aspects of parties directly involved in HRI $[94,96]$. To assess emotional and/or behavioral responses of candidates, audio and video observations are recorded and evaluated afterwards [89].

Objective performance measures quantify the output of HRI $[97,98]$. Related measures indicate how well an anthropomorphic robot supported the task completion by a human [89]. Sample task performance indicators include the efficiency and the speed of a performed task or the amount of errors occurred $[97,98]$.

Sensor data can be observational or analytical in nature. Some examples of observational sensors include microphones and cameras, which capture observations of the human partner during an interaction [99]. Additionally, perceiving a human's motion in order to react accordingly is an important aspect of HRI $[100,101]$. Such observational sensors can be used to understand the emotional state of the participant either from audio [102], video [3,103] or both [104, 105]. Emotions are "an episode of interrelated synchronized changes in the states of all or most of the five organismic subsystems in response to the evaluation of an external or internal stimulus event as relevant to major concerns of the organism" [106, p. 697] and are a major indicator for humans' well-being during HRI (see [12] for an overview). Besides their implication on human well-being during the HRI, robotic psychology has even shown that humans can catch artificial emotions from anthropomorphic robots [2] within an emotional contagion process (see [39] for an overview). Despite emotional expressions by robots, human emotions can be detected by sensors and inferred to the emotion of the person by trained models.

This "ability to encode an ensemble of sensory stimuli providing information about the emotional state of another individual" [107, p. 1] is referred to as emotion recognition. A major source of emotional recognition relies on humans' facial expression that has attracted considerable research interest for several years [108, 109]. Recently, deep learning algorithms have become popular. The pre-processed image is directly fed into deep networks to predict an output (see [3] for an overview), mainly relating to six universally known emotions (i.e., happy, sad, disgust, angry, fear, surprise) and sometimes including a neutral expression [109].

Another important means to gather information about human emotions during HRI is psycho-physiological data, such as electrodermal activity (EDA), electroencephalography (EEG), electrocardiogram (ECG), electromyography (EMG), and respiration (RESP) [88]. Among these signals, EDA, EEG and ECG are the most commonly applied indicators [110]. For example, in a stressful situation, the perspiration increases, affecting the conductivity of the skin and making the EDA signal rise. Emotions are related to the autonomic nervous system, which is accompanied by changes in physiological signals $[111,112]$. Consequently, physiological responses of participants can be used as indicators of human's arousal [90]. Wristbands, for instance, can conduct physiological data like Heart Rates, allowing assumptions regarding participant's stress level [113]. Physiological signals are difficult if not impossible to be controlled and therefore deceived [114]. Hence, using analytical sensor data leads to an objectification of data, since human participants generally are not able to manipulate their physiological reactions [88]. Observations and analytical sensors can be used to measure the emotions of an individual using audio [115], physiological [116] 
or visual signals [3, 117].

A literature review on the use of physiological signals in HRI to detect emotions points out that the baseline of the physiological signals should be gathered before recognizing the influence of the emotions [118, 119]. [120] indicates that the first encounter with a robot should take place before the experiment starts. That is certainly true if the impact of different behaviors of the robot is to be considered as emotional triggers rather than the first encounter. In the studies considered [118, 121-127] the experimental setup is divided into small subsections during HRI. These represent individual movements of the robot or tasks of the subject in the interaction with the robot. Within these sections, a certain emotional response of the subject is expected and later evaluated.

From a conceptual perspective research on emotions in HRI employs emotion models that abstract the subject's emotion to varying degrees (see [12] for an overview). The most popular model is Russels circumplex model [128]. The ground truth describing the subject's emotion in the individual sections is ensured by classifying the physiological signals, questionnaires, or adding further emotion recognition methods (such as facial recognition).

While extant research provides valuable insights on rather general evaluation criteria for human responses to robotic design, specific criteria for a responsible design of HRI are scarce. Specifically, criteria that capture ethical and legal aspects, such as human privacy, safety, and the transparency of the robotic design are scarce.

\section{Implications and Suggestions}

This paper addresses the state of the art from different disciplines (i.e., ethics, legal sciences, psychology, technology), relevant for the responsible design of HRI with anthropomorphic service robots which is pursued by "RoboTrust". Despite considerable progress achieved by research on responsible design of HRI, this review also reveals several limitations of previous empirical research through the lens of the particular disciplines. This Section therefore outlines four suggestions for continued research on the responsible design of HRI.

Extend the interdisciplinary perspective on responsible HRI. This overview has demonstrated the importance of an interdisciplinary approach, which is essential for the development and programming of responsible HRI with anthropomorphic service robots and for conducting and evaluating responsible HRI experiments. More research is needed that integrates different disciplines for a design of HRI which pays particular attention to human dignity, privacy, safety, and transparency.

Develop uniform standards to evaluate the responsible design of HRI. Most of the studies in this review have evaluated human responses to HRI based on self perceptions, third-rater perceptions or sensor data on emotional and/or behavioral measures (see Section 2.5). Future research should pay more attention to criteria that capture transparency, privacy, and security or human responses to these design parameters, such as trust and confidence.

Analyze longitudinal effects of responsible HRI. Instead of examining a single human experience in an HRI, future research should examine longitudinal effects of responsible robot design over time. This in turn might help to understand human responses to HRI beyond a first impression effect.

Use real-world environments to test the effects of responsible design of $H R I$. To date, still most studies on HRI have been conducted in laboratory settings (see Section 2.5). Design implications for responsible HRI would contribute to a deeper understanding of real-life and kind of "authentic" HRI.

\section{Acknowledgement}

This research was conducted as part of RoboTrust, a project of the Centre Responsible Digitality.

\section{References}

[1] M. Mara and M. Appel, "Science Fiction Reduces the Eeriness of Android Robots: A Field Experiment," Computers in Human Behavior, vol. 48, pp. 156-162, 2015.

[2] R. M. Stock and M. Merkle, "A Service Robot Acceptance Model: User Acceptance of Humanoid Robots During Service Encounters," in 2017 IEEE International Conference on Pervasive Computing and Communications Workshops, pp. 339-344, March 13-17, 2017.

[3] N. Rawal and R. M. Stock-Homburg, "Facial Emotion Expressions in Human-Robot Interaction: A Survey," To appear in International Journal of Social Robotics, 2021.

[4] M. Mende, M. L. Scott, J. van Doorn, D. Grewal, and I. Shanks, "Service Robots Rising: How Humanoid Robots Influence Service Experiences and Elicit Compensatory consumer responses," Journal of Marketing Research, vol. 56, no. 4, pp. 535-556, 2019.

[5] S. H. Ivanov, C. Webster, and K. Berezina, "Adoption of Robots and Service Automation by Tourism and Hospitality Companies," Revista Turismo \& Desenvolvimento, vol. 27, no. 28, pp. 1501-1517, 2017.

[6] M. Merkle, "Customer Responses to Service Robots-Comparing Human-Robot Interaction with Human-Human Interaction," in Proceedings of the 52nd Hawaii International Conference on System Sciences, pp. 1396-1405, January 8-11, 2019. 
[7] F. Schweitzer, R. Belk, W. Jordan, and M. Ortner, "Servant, Friend or Master? The Relationships Users Build with Voice-Controlled Smart Devices," Journal of Marketing Management, vol. 35, no. 7-8, pp. 693-715, 2019.

[8] D. Leyzberg, S. Spaulding, M. Toneva, and B. Scassellati, "The Physical Presence of a Robot Tutor Increases Cognitive Learning Gains," in Proceedings of the annual meeting of the cognitive science society, vol. 34, pp. 1882-1887, August 1-4, 2012.

[9] T. Fong, C. Thorpe, and C. Baur, Collaboration, Dialogue, Human-Robot Interaction, pp. 255-266. Berlin, Heidelberg, Germany: Springer, 2003.

[10] R. Belk, "Ethical Issues In Service Robotics and Artificial Intelligence," The Service Industries Journal, pp. 1-17, 2020.

[11] M. Knof, J. Heinisch, J. Kirchhoff, N. Rawal, K. David, O. von Stryk, and R. Stock-Homburg, "Implications from Responsible Human-Robot Interaction with Anthropomorphic Service Robots for Design Science," in Proceedings of the 55th Hawaii international conference on system sciences, January 3-7, 2022.

[12] R. Stock-Homburg, "Survey of Emotions in Human-Robot Interactions: Perspectives from Robotic Psychology on 20 Years of Research," International Journal of Social Robotics, pp. 1-23, 2021.

[13] M. Ahmad, O. Mubin, and J. Orlando, "A systematic review of adaptivity in human-robot interaction," Multimodal Technologies and Interaction, vol. 1, no. 3, p. 14, 2017.

[14] P. Baxter, J. Kennedy, E. Senft, S. Lemaignan, and T. Belpaeme, "From Characterising Three Years of HRI to Methodology and Reporting Recommendations," in 2016 11th ACM/IEEE International Conference on Human-Robot Interaction, pp. 391-398, 2016.

[15] R. Wullenkord and F. Eyssel, "Societal and ethical issues in hri," Current Robotics Reports, pp. 1-12, 2020.

[16] C. Lutz, M. Schöttler, and C. P. Hoffmann, "The privacy implications of social robots: Scoping review and expert interviews," Mobile Media \& Communication, vol. 7, no. 3, pp. 412-434, 2019.

[17] H. Zech, "Liability for AI: Public Policy Considerations," in ERA Forum, vol. 22, pp. 147-158, Springer, 2021.

[18] A. A. Sodemann, M. P. Ross, and B. J. Borghetti, "A Review of Anomaly Detection in Automated Surveillance," IEEE Transactions on Systems, Man, and Cybernetics, Part C (Applications and Reviews), vol. 42, no. 6, pp. 1257-1272, 2012.

[19] W. Oremus, "Terrifyingly Convenient: AI Assistants Can Give You the News, Order You a Pizza, and Tell You a Joke. All You have to do is Trust Them-Completely," 2016.

[20] M. Anderson and S. L. Anderson, "Machine Ethics: Creating an Ethical Intelligent Agent," in Machine Ethics and Robot Ethics, pp. 237-248, Routledge, 2020.

[21] G. D. Crnkovic and B. Çürüklü, "Robots: Ethical by Design," Ethics and Information Technology, vol. 14, no. 1, pp. 61-71, 2012.

[22] G. Veruggio and F. Operto, "The Debate on Roboethics," Philosophy \& Engineering, p. 23, 2007.
[23] The IEEE Global Initiative on Ethics of Autonomous and Intelligent Systems, "Ethically Aligned Design - A Vision for Prioritizing Human Well-being with Autonomous and Intelligent Systems," pp. 1-294, 2019.

[24] K. Abney, "Robotics, Ethical Theory, and Metaethics: A Guide for the Perplexed," Robot ethics: The ethical and social implications of robotics, pp. 35-52, 2012.

[25] S. Bringsjord and J. Taylor, "Introducing Divine-Command Robot Ethics," Robot ethics: the ethical and social implication of robotics, pp. 85-108, 2012.

[26] A. M. DeBaets, "Can a Robot Pursue the Good? Exploring Artificial Moral Agency," Journal of Evolution and Technology, vol. 24, no. 3, pp. 76-86, 2014.

[27] L. Floridi and J. W. Sanders, "On the Morality of Artificial Agents," Minds and machines, vol. 14, no. 3, pp. 349-379, 2004.

[28] J. Parthemore and B. Whitby, "What Makes Any Agent a Moral Agent? Reflections on Machine Consciousness and Moral Agency," International Journal of machine consciousness, vol. 5, no. 02, pp. 105-129, 2013.

[29] B. F. Malle, "Integrating Robot Ethics and Machine Morality: The Study and Design of Moral Competence in Robots," Ethics and Information Technology, vol. 18, no. 4, pp. 243-256, 2016.

[30] G. Veruggio, J. Solis, and M. Van der Loos, "Roboethics: Ethics Applied to Robotics [from the guest editors]," IEEE Robotics \& Automation Magazine, vol. 18, no. 1, pp. 21-22, 2011.

[31] P.-P. Verbeek, Moralizing Technology: Understanding and Designing the Morality of Things. University of Chicago Press, 2011.

[32] P.-P. Verbeek, "Some Misunderstandings About the Moral Significance of Technology," in The moral status of technical artefacts, pp. 75-88, Springer, 2014.

[33] S. L. Vargo and R. F. Lusch, "Service-Dominant Logic: Continuing the Evolution," Journal of the Academy of marketing Science, vol. 36, no. 1, pp. 1-10, 2008.

[34] N. Bostrom and E. Yudkowsky, "The Ethics of Artificial Intelligence," The Cambridge handbook of artificial intelligence, vol. 1, pp. 316-334, 2014.

[35] S. Du and C. Xie, "Paradoxes of Artificial Intelligence in Consumer Markets: Ethical Challenges and Opportunities," Journal of Business Research, vol. 129, pp. 961-974, 2021.

[36] B. Friedman and D. G. Hendry, Value Sensitive Design: Shaping Technology with Moral Imagination. Mit Press, 2019.

[37] G. Riva, R. M. Banos, C. Botella, B. K. Wiederhold, and A. Gaggioli, "Positive Technology: Using Interactive Technologies to Promote Positive Functioning," Cyberpsychology, Behavior, and Social Networking, vol. 15, no. 2, pp. 69-77, 2012.

[38] W. Wallach, "Robot Minds and Human Ethics: The Need for a Comprehensive Model of Moral Decision Making," Ethics and Information Technology, vol. 12, no. 3, pp. 243-250, 2010.

[39] R. Stock and M. A. Nguyen, "Robotic Psychology. What Do We Know About Human-Robot Interaction and What Do We Still Need to Learn?," in Proceedings of the 52nd Hawaii international conference on system sciences, 2019. 
[40] H. Matsuzaki and G. Lindemann, "The Autonomy-Safety-Paradox of Service Robotics in Europe and Japan: A Comparative Analysis," AI \& Society, vol. 31, no. 4, pp. 501-517, 2016.

[41] S. Loznen, C. Bolintineanu, and J. Swart, Electrical Product Compliance and Safety Engineering. Norwood, MA, USA: Artech House, 2017.

[42] R. van den Hoven van Genderen, "Privacy and Data Protection in the Age of Pervasive Technologies in AI and Robotics," European Data Protection Law Review, vol. 3, no. 3, pp. 338-352, 2017.

[43] W. Barfield, "Liability for Autonomous and Artificially Intelligent Robots," Paladyn, Journal of Behavioral Robotics, vol. 9, no. 1, pp. 193-203, 2018.

[44] F. P. Hubbard, "Sophisticated Robots: Balancing Liability, Regulation, and Innovation," Florida Law Review, vol. 66, no. 5, pp. 1803-1872, 2014.

[45] A. Lior, "AI Entities as AI agents: Artificial Intelligence Liability and the AI Respondeat Superior analogy," Mitchell Hamline Law Review, vol. 46, no. 5, pp. 1043-1102, 2020.

[46] O. J. Erdélyi and G. Erdélyi, "The AI Liability Puzzle and a Fund-Based Work-Around," Journal of Artificial Intelligence Research, vol. 70, pp. 1309-1334, 2021.

[47] R. Abbott, The Reasonable Robot: Artificial Intelligence and the Law. Cambridge, UK: Cambridge University Press, 2020.

[48] G. Wagner, "Robot Liability," in Liability for Artificial Intelligence and the Internet of Things (S. Lohsse, R. Schulze, and D. Staudenmeyer, eds.), pp. 27-62, Nomos, 2019.

[49] E. G. on Liability and N. T. N. T. Formation, "Liability for Artificial Intelligence and Other Emerging Digital Technologies," tech. rep., European Commission, 2019.

[50] E. Commission, "Proposal for a Regulation of the European Parliament and of the Council: Laying Down Harmonised Rules on Artificial Intelligence (Artificial Intelligence Act) and Amending Certain Union Legislative Acts," 2021.

[51] M. Rueben, A. Aroyo, C. Lutz, J. Schmolz, P. Van Cleynenbreugel, A. Corti, S. Agrawal, and W. Smart, "Themes and Research Directions in Privacy-Sensitive Robotics," in 2018 IEEE Workshop on Advanced Robotics and its Social Impacts, pp. 77-84, September 28-29, 2018.

[52] A. F. Westin, Privacy and Freedom. New York, NY: Atheneum, 1968.

[53] P. Hustinx, The Reform of EU Data Protection: Towards More Effective and More Consistent Data Protection across the EU, ch. 4, p. 62-72. Cambridge, UK: Cambridge University Press, 2014.

[54] O. Lynskey, "Deconstructing Data Protection: The 'Added-Value' of a Right to Data Protection in the EU Legal Order," International and Comparative Law Quarterly, vol. 63, no. 4, pp. 569-597, 2014.

[55] EU, "Regulation (EU) 2016/679 of the European Parliament and of the Council on the Protection of Natural Persons with Regard to the Processing of Personal Data and on the Free Movement of such Data, and Repealing Directive 95/46/EC (General Data Protection Regulation) (27 April 2016)."

[56] P. M. Schwartz, "Global Data Privacy: The EU Way," New York University Law Review, vol. 94, no. 1, pp. 771-818, 2019.
[57] C. Kuner, L. A. Bygrave, C. Docksey, and L. Drechsler, The EU General Data Protection Regulation (GDPR): A Commentary. Oxford, UK: Oxford University Press, 2020.

[58] T. Dreier and I. Spiecker genannt Döhmann, "Legal Aspects of Service Robotics," Poiesis \& Praxis, vol. 9, no. 3-4, pp. 201-217, 2012.

[59] E. Fosch-Villaronga, H. Felzmann, T. Mahler, and M. Ramos Montero, "Cloud Services for Robotic Nurses? Assessing Legal and Ethical Issues in the Use of Cloud Services for Healthcare Robots," in 2018 IEEE/RSJ International Conference on Intelligent Robots and Systems, pp. 290-296, October 1-5, 2018.

[60] T. Hoffmann and G. Prause, "On the Regulatory Framework for Last-Mile Robots," Machines, vol. 6, no. 3, 2018.

[61] E. Fosch Villaronga and C. Millard, "Cloud Robotics Law and Regulation," Queen Mary School of Law Legal Studies Research Paper, no. 295, 2018.

[62] H. Felzmann, E. Fosch-Villaronga, C. Lutz, and A. Tamo-Larrieux, "Robots and Transparency: The Multiple Dimensions of Transparency in the Context of Robot Technologies," IEEE Robotics \& Automation Magazine, vol. 26, no. 2, pp. 71-78, 2019.

[63] C. Lutz and A. Tamò, "RoboCode-Ethicists: Privacy-friendly Robots, An Ethical Responsibility of Engineers?," in Proceedings of the ACM Web Science Conference, pp. 1-12, June 28 - July 1, 2015.

[64] C. Lutz and A. Tamó-Larrieux, "The Robot Privacy Paradox: Understanding How Privacy Concerns Shape Intentions to Use Social Robots," Human-Machine Communication Journal, vol. 1, no. 1, pp. 87-111, 2020.

[65] E. Barnoviciu, V. Ghenescu, S.-V. Carata, M. Ghenescu, R. Mihaescu, and M. Chindea, "GDPR Compliance in Video Surveillance and Video Processing Application," in 2019 International Conference on Speech Technology and Human-Computer Dialogue (SpeD), pp. 1-6, October 10-12, 2019.

[66] A. Sidlauskas, "Video Surveillance and the GDPR," in Social Transformations in Contemporary Society 2019 (7), pp. 55-65, June 6-7, 2019.

[67] S. B. Barnes, "A Privacy Paradox: Social Networking in the United States," First Monday, 2006.

[68] P. A. Norberg, D. R. Horne, and D. A. Horne, "The Privacy Paradox: Personal Information Disclosure Intentions versus Behaviors," The Journal of Consumer Affairs, vol. 41, no. 1, pp. 100-126, 2007.

[69] R. Stock-Homburg and M. Hannig, "Is There a Privacy Paradox in the Workplace?," in Proceedings of the Forty-First International Conference on Information Systems, pp. 1-17, December 12-17, 2020.

[70] T. Kim and P. Hinds, "Who Should I Blame? Effects of Autonomy and Transparency on Attributions in Human-Robot Interaction," in ROMAN 2006-The 15th IEEE International Symposium on Robot and Human Interactive Communication, pp. 80-85, September 6-8, 2006.

[71] T. L. Sanders, T. Wixon, K. E. Schafer, J. Y. Chen, and P. Hancock, "The Influence of Modality and Transparency on Trust in Human-Robot Interaction," in 2014 IEEE International Inter-Disciplinary Conference on Cognitive Methods in Situation Awareness and Decision Support, pp. 156-159, March 3-6, 2014. 
[72] R. H. Wortham, A. Theodorou, and J. J. Bryson, "What Does the Robot Think? Transparency as a Fundamental Design Requirement for Intelligent Systems," in Proceedings of the IJCAI Workshop on Ethics for Artificial Intelligence, July 9, 2016.

[73] J. B. Lyons, G. G. Sadler, K. Koltai, H. Battiste, N. T. Ho, L. C. Hoffmann, D. Smith, W. Johnson, and R. Shively, "Shaping Trust Through Transparent Design: Theoretical and Experimental Guidelines," in Advances in human factors in robots and unmanned systems, pp. 127-136, Springer International Publishing Switzerland 2017, 2017.

[74] B. Nesset, D. A. Robb, J. Lopes, and H. Hastie, "Transparency in HRI: Trust and Decision Making in the Face of Robot Errors," in Companion of the 2021 ACM/IEEE International Conference on Human-Robot Interaction, pp. 313-317, March 9-11 2021.

[75] J. L. Wright, J. Y. Chen, and S. G. Lakhmani, "Agent Transparency and Reliability in Human-Robot Interaction: The Influence on User Confidence and Perceived Reliability," IEEE Transactions on Human-Machine Systems, vol. 50, no. 3, pp. 254-263, 2020.

[76] J. Y. Chen, K. Procci, M. Boyce, J. Wright, A. Garcia, and M. Barnes, "Situation Awareness-Based Agent Transparency," Tech. Rep. ARL-TR-6905, Aberdeen Proving Ground (MD): Army Research Laboratory (US), April 2014.

[77] J. Lyons, "Being Transparent about Transparency: A Model for Human-Robot Interaction," Tech. Rep. SS-13-07, AAAI Spring Symposium Series, March 2013.

[78] M. R. Endsley, "Toward a Theory of Situation Awareness in Dynamic Systems," Human Factors, vol. 37, no. 1, pp. 32-64, 1995.

[79] A. S. Rao and M. P. Georgeff, "BDI Agents: From Theory to Practice," in Proceedings of the First International Conference on Multiagent Systems, pp. 312-319, June 12-14, 1995.

[80] J. D. Lee and K. A. See, "Trust in Automation: Designing for Appropriate Reliance," Human Factors, vol. 46, no. 1, pp. 50-80, 2004.

[81] A. Bhaskara, M. Skinner, and S. Loft, "Agent Transparency: A Review of Current Theory and Evidence," IEEE Transactions on Human-Machine Systems, vol. 50, no. 3, pp. 215-224, 2020.

[82] F. Rajabiyazdi and G. A. Jamieson, "A Review of Transparency (seeing-into) Models," in 2020 IEEE International Conference on Systems, Man, and Cybernetics, pp. 302-308, October 11-14, 2020.

[83] A. Weller, "Transparency: Motivations and Challenges," in Proceedings of the 2017 ICML Workshop on Human Interpretability in Machine Learning, pp. 55-62, August 10, 2017.

[84] IEEE Standards Association, "IEEE Draft Standard for Transparency of Autonomous Systems," IEEE P7001/D1, pp. 1-70, June 2020.

[85] D. Thomas, Middleware for Efficient Programming of Autonomous Mobile Robots. $\mathrm{PhD}$ thesis, Technische Universität, Darmstadt, November 2010.

[86] K. Fischer, "When Transparent Does Not Mean Explainable," in Proceedings of Workshop on Explainable Robotic Systems in conjunc with the HRI 2018 Conference, March 5, 2018.
[87] M. Scriven, Evaluation Thesaurus. California, US: Sage, 1981.

[88] C. L. Bethel, K. Salomon, R. R. Murphy, and J. L. Burke, "Survey of Psychophysiology Measurements Applied to Human-Robot Interaction," in RO-MAN 2007-The 16th IEEE International Symposium on Robot and Human Interactive Communication, pp. 732-737, August 26-29, 2007.

[89] C. L. Bethel and R. R. Murphy, "Review of Human Studies Methods in HRI and Recommendations," International Journal of Social Robotics, vol. 2, no. 4, pp. 347-359, 2010.

[90] N. Homburg, "How to Include Humanoid Robots into Experimental Research: A Multi-Step Approach," in Proceedings of the 51st Hawaii International Conference on System Sciences, pp. 4423-4432, January 2-6, 2018.

[91] L. Tiberio, A. Cesta, and M. Olivetti Belardinelli, "Psychophysiological Methods to Evaluate User's Response in Human Robot Interaction: A Review and Feasibility Study," Robotics, vol. 2, no. 2, pp. 92-121, 2013.

[92] C. de Jong, R. Kühne, J. Peter, C. L. van Straten, and A. Barco, "Intentional Acceptance of Social Robots: Development and Validation of a Self-Report Measure for Children," International Journal of Human-Computer Studies, vol. 139, p. 102426, 2020.

[93] A. Steinfeld, T. Fong, D. Kaber, M. Lewis, J. Scholtz, A. Schultz, and M. Goodrich, "Common Metrics for Human-Robot Interaction," in Proceedings of the 1st ACM SIGCHI/SIGART conference on Human-robot interaction, pp. 33-40, March 2-3, 2006.

[94] J. Li, W. Ju, and C. Nass, "Observer Perception of Dominance and Mirroring Behavior in Human-Robot Relationships," in 2015 10th ACM/IEEE International Conference on Human-Robot Interaction, pp. 133-140, March 2-5, 2015.

[95] K. E. Schaefer, Measuring Trust in Human Robot Interactions: Development of the "Trust Perception Scale-HRI”, ch. 10, pp. 191-218. Boston, MA: Springer, 2016.

[96] A. K. Coyne, A. Murtagh, and C. McGinn, "Using the Geneva Emotion Wheel to Measure Perceived Affect in Human-Robot Interaction," in Proceedings of the 2020 ACM/IEEE International Conference on Human-Robot Interaction, pp. 491-498, March 24-26, 2020.

[97] H. Admoni, T. Weng, B. Hayes, and B. Scassellati, "Robot Nonverbal Behavior Improves Task Performance in Difficult Collaborations," in 2016 11th ACM/IEEE International Conference on Human-Robot Interaction, pp. 51-58, 2016.

[98] R. R. Murphy and D. Schreckenghost, "Survey of Metrics for Human-Robot Interaction," in 2013 8th ACM/IEEE International Conference on Human-Robot Interaction (HRI), pp. 197-198, March 3-6, 2013.

[99] R. Stiefelhagen, C. Fugen, R. Gieselmann, H. Holzapfel, K. Nickel, and A. Waibel, "Natural Human-Robot Interaction Using Speech, Head Pose and Gestures," in 2004 IEEE/RSJ International Conference on Intelligent Robots and Systems, vol. 3, pp. 2422-2427, September 28, - October 2, 2004.

[100] J. Bütepage, H. Kjellström, and D. Kragic, "Anticipating Many Futures: Online Human Motion Prediction and Generation for Human-Robot Interaction," in 2018 IEEE international conference on robotics and automation, pp. 4563-4570, IEEE, 2018. 
[101] N. Jarrassé, J. Paik, V. Pasqui, and G. Morel, "How Can Human Motion Prediction Increase Transparency?," in 2008 IEEE International Conference on Robotics and Automation, pp. 2134-2139, IEEE, 2008.

[102] J. G. Rázuri, D. Sundgren, R. Rahmani, A. Moran, I. Bonet, and A. Larsson, "Speech Emotion Recognition in Emotional Feedback for Human-Robot Interaction," International Journal of Advanced Research in Artificial Intelligence, vol. 4, no. 2, pp. 20-27, 2015.

[103] P. P. Filntisis, N. Efthymiou, G. Potamianos, and P. Maragos, "Emotion Understanding in Videos Through Body, Context, and Visual-Semantic Embedding Loss," in European Conference on Computer Vision, pp. 747-755, Springer, 2020.

[104] I. Kansizoglou, L. Bampis, and A. Gasteratos, "An Active Learning Paradigm for Online Audio-Visual Emotion Recognition," IEEE Transactions on Affective Computing, 2019.

[105] E. Avots, T. Sapiński, M. Bachmann, and D. Kamińska, "Audiovisual Emotion Recognition in Wild," Machine Vision and Applications, vol. 30, no. 5, pp. 975-985, 2019.

[106] K. R. Scherer, "What Are Emotions? And How Can They Be Measured?," Social Science Information, vol. 44, no. 4, pp. 695-729, 2005.

[107] V. Ferretti and F. Papaleo, "Understanding Others: Emotion Recognition in Humans and Other Animals," Genes, Brain and Behavior, vol. 18, no. 1, p. e12544, 2019.

[108] D. Canedo and A. Neves, "Facial Expression Recognition Using Computer Vision: A Systematic Review," Applied Sciences, vol. 9, 112019.

[109] S. Li and W. Deng, "Deep Facial Expression Recognition: A Survey," IEEE Transactions on Affective Computing, pp. 1-1, 2020.

[110] S. Greene, H. Thapliyal, and A. Caban-Holt, "A Survey of Affective Computing for Stress Detection: Evaluating technologies in stress detection for better health," IEEE Consumer Electronics Magazine, vol. 5, no. 4, pp. 44-56, 2016.

[111] R. W. Levenson, Emotion and The Autonomic Nervous System: A Prospectus for Research on Autonomic Specificity., ch. xiii, pp. 17-42. Oxford, England: John Wiley \& Sons, 1988.

[112] S. D. Kreibig, "Autonomic Nervous System Activity in Emotion: A Review," Biological Psychology, vol. 84, no. 3, pp. 394-421, 2010.

[113] S. Ollander, C. Godin, A. Campagne, and S. Charbonnier, "A Comparison of Wearable and Stationary Sensors for Stress Detection," in 2016 IEEE International Conference on Systems, Man, and Cybernetics, pp. 004362-004366, October 9-12, 2016.

[114] H. Gunes and M. Pantic, "Automatic, Dimensional and Continuous Emotion Recognition," International Journal of Synthetic Emotions, vol. 1, no. 1, pp. 68-99, 2010.

[115] S. Yoon, S. Byun, and K. Jung, "Multimodal Speech Emotion Recognition Using Audio and Text," in 2018 IEEE Spoken Language Technology Workshop, pp. 112-118, IEEE, 2018.

[116] L. Shu, J. Xie, M. Yang, Z. Li, Z. Li, D. Liao, X. Xu, and X. Yang, "A Review of Emotion Recognition Using Physiological Signals," Sensors, vol. 18, no. 7, p. 2074 2018.
[117] L.-N. Do, H.-J. Yang, H.-D. Nguyen, S.-H. Kim, G.-S. Lee, and I.-S. Na, "Deep Neural Network-Based Fusion, Model for Emotion Recognition Using Visual Data," The Journal of Supercomputing, pp. 1-18, 2021.

[118] M. Val-Calvo, J. R. Alvarez-Sanchez, J. M. Ferrandez-Vicente, and E. Fernandez, "Affective Robot Story-Telling Human-Robot Interaction: Exploratory Real-Time Emotion Estimation Analysis Using Facial Expressions and Physiological Signals," IEEE Access, vol. 8, pp. 134051-134066, 2020.

[119] C. Liu, P. Rani, and N. Sarkar, "Human-Robot Interaction Using Affective Cues," in ROMAN 2006 - The 15th IEEE International Symposium on Robot and Human Interactive Communication, pp. 285-290, September 2006.

[120] C. D. Kidd and C. Breazeal, "Human-Robot Interaction Experiments: Lessons Learned," in Proceedings of the Symposium on Robot Companions: Hard Problems and Open Challenges in Robot-Human Interaction, vol. 5, pp. 141-142, April 2005.

[121] D. Kulic and E. Croft, "Anxiety Detection during Human-Robot Interaction," in 2005 IEEE/RSJ International Conference on Intelligent Robots and Systems, pp. 616-621, August 2005.

[122] R. Kagawa, M. Nobuto, Y. Someya, R. Yoshida, and M. Sugaya, "Affect Evaluation of Biological Information Approached by a Nursing/care Robot," in Proceedings of the Asia Pacific Conference on Robot IoT System Development and Platform, pp. 28-31, October 30 - November 2018.

[123] S. Suzuki, M. N. A. M. Anuardi, P. Sripian, N. Matsuhira, and M. Sugaya, "Multi-user Robot Impression with a Virtual Agent and Features Modification According to Real-time Emotion from Physiological Signals," in 2020 29th IEEE International Conference on Robot and Human Interactive Communication, pp. 1006-1012, August 2020.

[124] Y. Mohammad and T. Nishida, "Using Physiological Signals to Detect Natural Interactive Behavior," Applied Intelligence, vol. 33, no. 1, pp. 79-92, 2010.

[125] A. M. Zanchettin, L. Bascetta, and P. Rocco, "Acceptability of Robotic Manipulators in Shared Working Environments through Human-Like Redundancy Resolution," Applied Ergonomics, vol. 44, no. 6, pp. 982-989, 2013.

[126] P. Rani and N. Sarkar, "Making Robots Emotion-Sensitive - Preliminary Experiments and Results," in ROMAN 2005. IEEE International Workshop on Robot and Human Interactive Communication, 2005., pp. 1-6, August 2005.

[127] P. Rani and N. Sarkar, "Operator Engagement Detection and Robot Behavior Adaptation in Human-Robot Interaction," in Proceedings of the 2005 IEEE International Conference on Robotics and Automation, pp. 2051-2056, April 2005.

[128] J. A. Russell, "A Circumplex Model of Affect.," Journal of personality and social psychology, vol. 39, no. 6, pp. 1161-1178, 1980. Publisher: American Psychological Association. 